\title{
Walking speed and subclinical atherosclerosis in healthy older adults: the Whitehall II study
}

\author{
Mark Hamer, ${ }^{1}$ Mika Kivimaki, ${ }^{1}$ Avijit Lahiri, ${ }^{2}$ Ajay Yerramasu, ${ }^{2}$ John E Deanfield, ${ }^{3}$ \\ Michael G Marmot, ${ }^{1}$ Andrew Steptoe ${ }^{1}$
}

${ }^{1}$ Department of Epidemiology and Public Health, University College London, London, UK ${ }^{2}$ Cardiac Imaging and Research Centre, Wellington Hospital, London, UK ${ }^{3}$ Vascular Physiology Unit, Institute of Child Health, University College London, London, UK

\section{Correspondence to}

Dr Mark Hamer, Department of Epidemiology and Public Health, 1-19 Torrington Place, University College London, London WC1E 6BT, UK; m.hamer@ucl.ac.uk

Accepted 10 November 2009

This paper is freely available online under the BMJ Journals unlocked scheme, see http:// heart.bmi.com/site/about/ unlocked.xhtml.

\section{ABSTRACT}

Objective Extended walking speed is a predictor of incident cardiovascular disease (CVD) in older individuals, but the ability of an objective short-distance walking speed test to stratify the severity of preclinical conditions remains unclear. This study examined whether performance in an 8-ft walking speed test is associated with metabolic risk factors and subclinical atherosclerosis.

Design Cross-sectional.

Setting Epidemiological cohort.

Participants 530 adults laged $63 \pm 6$ years, 50.3\% male) from the Whitehall II cohort study with no known history or objective signs of CVD.

Main outcome Electron beam computed tomography and ultrasound was used to assess the presence and extent of coronary artery calcification (CAC) and carotid intima-media thickness (IMT), respectively.

Results High levels of CAC (Agatston score $>100$ ) were detected in $24 \%$ of the sample; the mean IMT was $0.75 \mathrm{~mm}$ (SD 0.15). Participants with no detectable CAC completed the walking course $0.16 \mathrm{~s}(95 \% \mathrm{Cl} 0.04$ to 0.28 ) faster than those with $C A C \geq 400$. Objectively assessed, but not self-reported, faster walking speed was associated with a lower risk of high CAC lodds ratio $0.62,95 \% \mathrm{Cl} 0.40$ to 0.96$)$ and lower IMT ( $\beta=-0.04$, $95 \% \mathrm{Cl}-0.01$ to $-0.07 \mathrm{~mm}$ ) in comparison with the slowest walkers (bottom third), after adjusting for conventional risk factors. Faster walking speed was also associated with lower adiposity, C-reactive protein and low-density lipoprotein cholesterol.

Conclusions Short-distance walking speed is associated with metabolic risk and subclinical atherosclerosis in older adults without overt CVD. These data suggest that a non-aerobically challenging walking test reflects the presence of underlying vascular disease.

Performance on extended walking tests predicts total mortality and incident cardiovascular disease (CVD) in older individuals, ${ }^{1}$ but the ability of shortdistance walking speed to stratify the severity of subclinical CVD remains uncertain. Several noninvasive imaging techniques can be employed to examine the extent of subclinical atherosclerosis, ${ }^{2} 3$ which precedes CVD. In a study of 387 healthy, older participants from the Cardiovascular Health Study, coronary artery calcification (CAC) was inversely related to objectively measured walking speed in women but such an association was not seen in men. ${ }^{4}$ In the Three-City study, carotid intima-media thickness (IMT), another proxy measure of subclinical atherosclerosis, was inversely associated with objectively measured maximum walking speed. ${ }^{5}$ In the Multi-Ethnic Study of Atherosclerosis self-reported brisk walking pace was associated with lower CAC and IMT in men but only IMT in women. ${ }^{6}$

Short-distance tests of walking speed compared with those of long-distance and maximum walking speed have several advantages. They can be performed using relatively little clinic space, they are particularly safe and easy to administer, and are not aerobically demanding for the participant. We are not aware of any large-scale studies that have examined the ability of a short-distance walking speed test to capture variation in multiple markers of preclinical atherosclerosis. The aim of the present study was therefore to examine whether the performance in an 8-ft walking speed test is associated with metabolic risk factors and subclinical atherosclerosis, assessed from both CAC and carotid IMT among older men and women with no overt CVD. A second aim was to compare the predictive utility of the objective walking test with that of self-reported walking pace.

\section{METHODS}

\section{Participants}

A subsample of participants from phase 7 (2003-4) of the Whitehall II epidemiological cohort ${ }^{7}$ were recruited for the assessment of CAC in 2006-8. The inclusion criteria for the study were no history or objective signs of coronary heart disease, verified by clinical examination including ECG, and no previous diagnosis or treatment for hypertension or diabetes, inflammatory diseases (including arthritis), or allergies. Thirteen participants $(2.4 \%)$ were prescribed lipid-lowering medication (statins). Volunteers were of white European origin, aged $53-76$ years, and $56.5 \%$ were in full-time employment. Selection was stratified by grade of employment (current or most recent) to include higher and lower socioeconomic status participants. From the initially invited 1169 participants, 323 (27.6\%) were not eligible (mainly because of prescribed antihypertensive medications) and $303(25.9 \%)$ declined to take part. A higher proportion of participants from lower work grades declined to take part compared with higher grades, (38.6\% vs $20.3 \%$, respectively). Participants were prohibited from using any antihistamine or anti-inflammatory medication 7 days before testing, and were rescheduled if they reported colds or other infections on the day of testing. Participants gave full informed consent to participate in the study and ethical approval was obtained from the UCLH committee on the ethics of human research. 


\section{Clinical assessment}

Details of the Whitehall II phase 7 clinical assessment are published elsewhere. ${ }^{7}$ Walking speed was measured by a trained nurse over a clearly marked 8-ft walking course using a standardised protocol. ${ }^{8}$ Participants wore either low-heeled closefitting footwear or walked barefoot. Before the test, participants were shown the walking course and asked to "walk to the other end of the course at your usual walking pace, just as if you were walking down the street to go the shops. Walk all the way past the other end of the tape before you stop.' The starting position was standing with both feet together at the start of the course. Participants were asked to begin walking when properly positioned. The stopwatch was started as the participant's foot hit the floor across the starting line. Nurses walked behind and to the side of the participant and stopped timing when the participant's foot hit the floor after the end of the walking course. The test was repeated three times, and performance time on the last walk was used for the present analyses. We also calculated coefficient of variation of the three walks for each participant. Participants reported habitual physical activity from a 20 -item questionnaire, ${ }^{9}$ a question about usual walking pace (slow/average/brisk/ fast), current smoking levels, weekly alcohol intake (units per week) and self-rated health (excellent/very good/good/fair/poor). The physical function scale from the medical outcomes study short-form 36 health survey (SF-36) was used to assess activities of daily living. ${ }^{10}$ Respondents were asked about limitations to everyday activities such as lifting, climbing stairs and walking. Scores can range from 0 to 100 , with lower scores indicating greater impairment. Anthropometric measures were recorded in light clothing for the calculation of body mass index (BMI), and body fat percentage was measured using a bio-impedance device (BodyStat 1500 Medical, UK). Fasting venous blood samples were taken for the analysis of C-reactive protein (CRP), which was performed using high-sensitivity ELISA (R\&D Systems, Oxford, UK), and total and high-density lipoprotein (HDL) cholesterol and triglycerides, which were measured within $72 \mathrm{~h}$ in serum stored at $4^{\circ} \mathrm{C}$ using enzymatic colorimetric methods. Low-density lipoprotein (LDL) cholesterol was derived using the Friedewald equation. Glucose homeostasis was assessed from fasting glucose.

\section{Markers of subclinical atherosclerosis}

The assessment of CAC was performed using electron beam computed tomography (GE Imatron C-150; San Francisco, California, USA) as previously described. ${ }^{11}$ In brief, 40 contiguous $3-\mathrm{mm}$ slices were obtained during a single breath-hold starting at the carina and proceeding to the level of the diaphragm. Scan time was $100 \mathrm{~ms} /$ slice, synchronised to $40 \%$ of the $\mathrm{R}-\mathrm{R}$ interval. Agatston and volumetric calcium scores were calculated to quantify the extent of CAC by a single experienced investigator blinded to the clinical data on an Aquarius workstation (TeraRecon Inc, San Mateo, California, USA). Carotid IMT was measured using ultrasound scanning. ${ }^{3}$ The right and left common carotid arteries were scanned with a $5-10-\mathrm{MHz}$ probe (Alokia Prosound 2000, Tokyo, Japan). The carotid bulb was identified, and longitudinal two-dimensional ultrasonographic images of the common carotid artery $1-2 \mathrm{~cm}$ proximal to the carotid bulb were obtained. The optimal longitudinal image was acquired on the R-wave of the ECG and continuously recorded on video tape for $5 \mathrm{~s}$. Measurements of the posterior wall of the artery were made from stored images with electronic calipers. Carotid IMTwas calculated as the distance between the first bright light (lumen-intima interface) and the leading edge of the second bright light (media-adventitia interface). The three maximum measures from the right carotid artery in three different frames and the three maximum measures from the left common carotid artery in three different frames were averaged.

\section{Statistical analysis}

Thirteen participants with missing data ( $\mathrm{n}=6$ walking test, $\mathrm{n}=7$ bloods) were excluded from all analyses. Walking speed was stratified into three equal sex-specific groups, denoting fast $(<1.73 \mathrm{~s})$, medium (women, 1.73-2.10 s; men, 1.73-1.94 s) and slow (women $>2.10 \mathrm{~s}$, men $>1.94 \mathrm{~s}$ ). Walking speed variability was also stratified into three equal groups, denoting low $(<5 \%)$, medium (5-8\%), high (>8\%). Because of the skewed distribution, CAC was dichotomised and multivariate logistic regression analyses were employed to examine the association of walking speed and variability with the presence of high CAC (Agatston score $\geq 100$ ). This threshold was based on previous data that demonstrated maximum sensitivity and specificity for detecting cardiovascular events at a threshold calcium score of 100 or greater. ${ }^{12}$ We calculated odds ratios (OR) and 95\% CI for the risk of high CAC according to objectively measured and self-reported walking speed categories, adjusting for age, sex, employment grade (as a marker of social position), smoking, SF-36, statins and conventional risk factors, including BMI, systolic blood pressure, LDL and HDL cholesterol, fasting glucose and CRP. Since the majority of participants reported 'average' or 'brisk' walking pace we constructed two categories consisting of 'slow/average' and 'brisk/fast' pace. For consistency the objectively measured walking speed was also split into two categories for these analyses, representing the bottom third (slowest) and upper twothirds (medium/fast pace). We employed general linear models to examine associations of walking speed with carotid IMT, making the same adjustments as stated above. General linear models were also employed to examine associations of walking speed with metabolic risk markers, including BMI and body fat, systolic blood pressure, total, HDL and LDL cholesterol, logtransformed CRP and fasting glucose. All analyses were conducted using SPSS version 15.

\section{RESULTS}

The sample consisted of 530 participants (284 men, 246 women, mean $\pm S D$ age $63 \pm 6$ years). Participants had moderately raised cholesterol, but generally demonstrated low risk factors with low rates of smoking and obesity and normal blood pressure (table 1). None of the participants rated their health as 'poor', with $68 \%$ of the sample giving ratings of 'excellent' or 'very good'. The time taken to complete the walking test ranged from 1.0 to $3.5 \mathrm{~s}$ and the average coefficient of variation between tests was $6.7 \%$. Faster walkers were slightly younger, more likely to come from higher work grades, record higher scores on the SF-36 (better physical function) and report better health (table 1). In addition, faster walking speed was associated with lower adiposity, CRP and LDL cholesterol after adjusting for age (see table 1). Blood pressure levels and fasting glucose were unrelated to walking speed. Participants who reported a usual walking pace of 'brisk/ fast' demonstrated faster walking times (age and sex-adjusted mean difference $-0.15,95 \% \mathrm{CI}-0.21$ to $-0.08 \mathrm{~s}$ ) compared with those reporting 'slow/average' walking pace. In addition, participants with higher self-reported physical health (top third of SF-36 scores) had marginally faster walking times (age and sexadjusted mean difference $-0.07,95 \% \mathrm{CI}-0.15$ to $0.01 \mathrm{~s}$ ) than those in the lowest third.

High levels of CAC (Agatston score $\geq 100$ ) were detected in $24 \%$ of the sample. The association between self-reported and objectively assessed walking pace is shown in table 2. Objectively 
Table 1 Characteristics of the sample in relation to sex specific walking speed groups (mean \pm SD)

\begin{tabular}{|c|c|c|c|c|}
\hline Variable & All $(n=530)$ & Slowest $(n=188$ ) & Medium $(n=180)$ & Fastest $(n=162)$ \\
\hline Age (years) & $62.8 \pm 5.7$ & $63.7 \pm 5.8$ & $62.7 \pm 5.7$ & $62.4 \pm 5.3 \dagger$ \\
\hline Work grade (\% lower grades) & 24.2 & 26.1 & 24.4 & $15.4 \dagger$ \\
\hline Marital status ( $\%$ married) & 63.5 & 62.6 & 68.0 & 62.7 \\
\hline Current smokers (\%) & 6.2 & 6.4 & 4.4 & 5.6 \\
\hline Alcohol (units/week) & $9.0 \pm 8.6$ & $9.8 \pm 9.9$ & $9.2 \pm 9.2$ & $8.3 \pm 7.5$ \\
\hline Prescribed lipid-lowering medication (\%) & 2.4 & 3.2 & 3.3 & 0.6 \\
\hline SF-36 physical function score & $82.8 \pm 14.0$ & $80.3 \pm 15.0$ & $84.0 \pm 13.5 \ddagger$ & $84.3 \pm 12.8 \neq$ \\
\hline Self-rated health (\% fair) & 4.7 & 6.4 & 5.0 & $2.5 \dagger$ \\
\hline Moderate-vigorous activity (MET h/week)* & $34.5 \pm 29.6$ & $32.6 \pm 27.4$ & $35.6 \pm 26.4$ & $35.6 \pm 34.6$ \\
\hline Systolic blood pressure (mm Hg) & $123.2 \pm 16.6$ & $125.6 \pm 17.1$ & $122.8 \pm 16.9$ & $123.5 \pm 14.9$ \\
\hline Body mass index $\left(\mathrm{kg} / \mathrm{m}^{2}\right)$ & $25.6 \pm 4.1$ & $26.2 \pm 4.1$ & $25.7 \pm 3.6$ & $25.6 \pm 3.9$ \\
\hline Body fat $\%$ & $30.8 \pm 8.3$ & $31.4 \pm 0.4$ & $30.7 \pm 0.4$ & $30.1 \pm 0.4 \neq$ \\
\hline Total cholesterol $(\mathrm{mmol} / \mathrm{l})$ & $5.89 \pm 0.94$ & $5.88 \pm 0.97$ & $5.97 \pm 1.00$ & $5.72 \pm 1.01$ \\
\hline HDL cholesterol (mmol/l) & $1.72 \pm 0.48$ & $1.68 \pm 0.47$ & $1.71 \pm 0.44$ & $1.71 \pm 0.50$ \\
\hline LDL cholesterol (mmol/l) & $3.91 \pm 0.95$ & $3.95 \pm 0.93$ & $4.00 \pm 0.97$ & $3.76 \pm 0.94 \ddagger$ \\
\hline Log C-reactive protein & $2.44 \pm 0.14$ & $2.46 \pm 0.16$ & $2.43 \pm 0.13$ & $2.43 \pm 0.14 \neq$ \\
\hline Fasting glucose $(\mathrm{mmol} / \mathrm{l})$ & $5.18 \pm 0.51$ & $5.16 \pm 0.56$ & $5.18 \pm 0.49$ & $5.21 \pm 0.45$ \\
\hline
\end{tabular}

* Metabolic equivalents (MET) defined from moderate (3-6 MET) to vigorous (>6 MET) physical activity.

+Significant difference compared with slowest walkers.

$¥$ Significant difference compared with slowest walkers in age-adjusted analyses.

HDL, high-density lipoprotein; LDL, low-density lipoprotein; SF-36, short-form 36.

assessed (but not self-reported) faster walking speed was associated with a lower risk of high CAC after adjustment for potential confounders, OR 0.62 (95\% CI 0.40 to 0.96 ) for the fastest (upper two-thirds) versus slowest (bottom third) walkers. Using linear analyses there was a marginal association between the log-transformed Agatston score and continuous walking time (age and sex adjusted $\beta=0.49,95 \% \mathrm{CI}-0.04$ to 1.02 ), indicating a greater extent of CAC in slower walkers. On average, there was a difference of $0.16 \mathrm{~s}$ (95\% CI 0.04 to 0.28 ) in the time to complete the walking course in participants with no detectable CAC compared with those with CAC of 400 or greater (see figure 1). Objectively assessed faster walking speed was also associated with lower IMT $(\beta=-0.034,95 \% \mathrm{CI}-0.002$ to $-0.067 \mathrm{~mm})$ in comparison with the slowest walkers (bottom third), after adjusting for age, sex, social status, smoking, SF-36 and conventional risk factors (figure 2). No sex differences were observed in these associations (eg, $\mathrm{p}=0.64$ for sex interaction with walking speed and CAC). High walking variability (top third) was not a significant predictor of CAC (age and sex adjusted OR $1.28,95 \%$ CI 0.76 to 2.18 ) or IMT ( $\beta=-0.008,95 \%$ $\mathrm{CI}-0.041$ to $0.025 \mathrm{~mm}$ ) in comparison with low walking variability (bottom third).

Table 2 Logistic regression of objective and self-reported walking pace on risk of CAC of 100 or greater

\begin{tabular}{|c|c|c|c|c|}
\hline $\begin{array}{l}\text { Walking } \\
\text { pace }\end{array}$ & $\mathbf{N}$ & $\begin{array}{l}\text { Model } 1 \\
\text { OR (95\% CI) }\end{array}$ & $\begin{array}{l}\text { Model } 2 \\
\text { OR }(95 \% \mathrm{CI})\end{array}$ & $\begin{array}{l}\text { Model } 3 \\
\text { OR (95\% CI) }\end{array}$ \\
\hline \multicolumn{5}{|l|}{ Objective } \\
\hline Slowest ${ }^{*}$ & 188 & 1.00 & 1.00 & 1.00 \\
\hline Brisk/fast & 342 & $0.61(0.40$ to 0.92$)$ & $0.61(0.40$ to 0.94$)$ & $0.62(0.40$ to 0.96$)$ \\
\hline $\mathrm{p}$ Value & & 0.01 & 0.03 & 0.03 \\
\hline \multicolumn{5}{|l|}{ Self-reported } \\
\hline Slow/average & 315 & 1.00 & 1.00 & 1.00 \\
\hline Brisk/fast & 213 & $0.84(0.54$ to 1.31$)$ & $0.88(0.56$ to 1.39$)$ & $0.93(0.58$ to 1.50$)$ \\
\hline p Value & & 0.444 & 0.589 & 0.773 \\
\hline
\end{tabular}

Model 1 adjusted for age and sex.

Model 2 with further adjustment for employment grade and smoking.

Model 3 with further adjustment for systolic blood pressure, low-density lipoprotein and

high-density lipoprotein cholesterol, C-reactive protein, fasting glucose, body mass index, short-form 36 score, lipid-lowering medication.

*Slowest third consists of women with walking time greater than $2.10 \mathrm{~s}$ and men greater than $1.94 \mathrm{~s}$.

$\mathrm{CAC}$, coronary artery calcification; $\mathrm{OR}$, odds ratio.

\section{DISCUSSION}

The present study examined the association of walking speed with markers of subclinical atherosclerosis, assessed from both CAC and carotid IMT, in a sample of older adults without overt CVD. Faster pace walking speed assessed with a short-distance test was associated with a lower prevalence and extent of CAC and lower IMT values, independently of age and other CVD risk factors. These associations were not observed when using the self-reported measure of walking pace. In a recent analysis of the full Whitehall II cohort that employed the short-distance test, age-adjusted walking speed was approximately 13\% higher in the highest employment grade compared with the lowest, ${ }^{13}$ although our findings suggest that associations with subclinical atherosclerosis are independent of social status. We also observed an association between walking speed and several markers of metabolic health. Replicable findings across two independent measures of preclinical atherosclerosis and consistent associations with atherosclerosis risk factors suggest that the association between walking speed and preclinical atherosclerosis was robust.

CAC has been shown to be independently predictive of $\mathrm{CHD}$ risk over and above traditional cardiac risk factors, ${ }^{2}$ and thus may be important in the early detection of atherosclerosis. Our findings extend those reported in the few previous smaller-scale

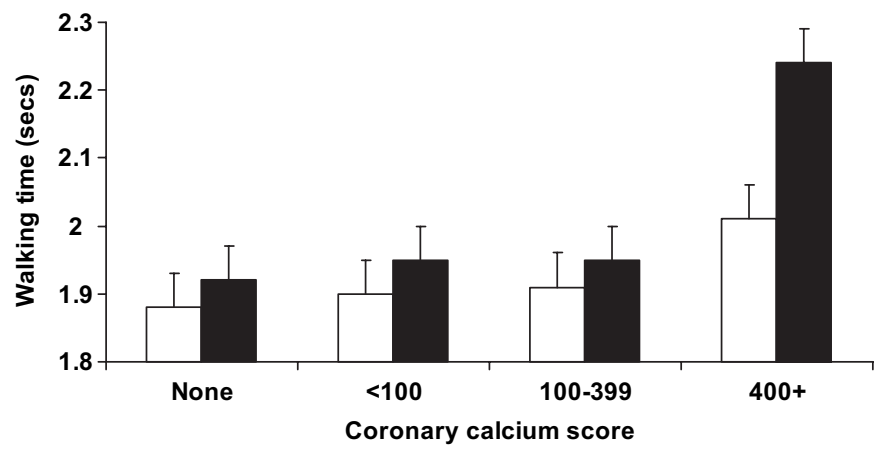

Figure 1 Time to complete walking test in relation to coronary artery calcium scores. Values are mean \pm SEM, adjusted for age. White bars represent men, filled bars represent women. 


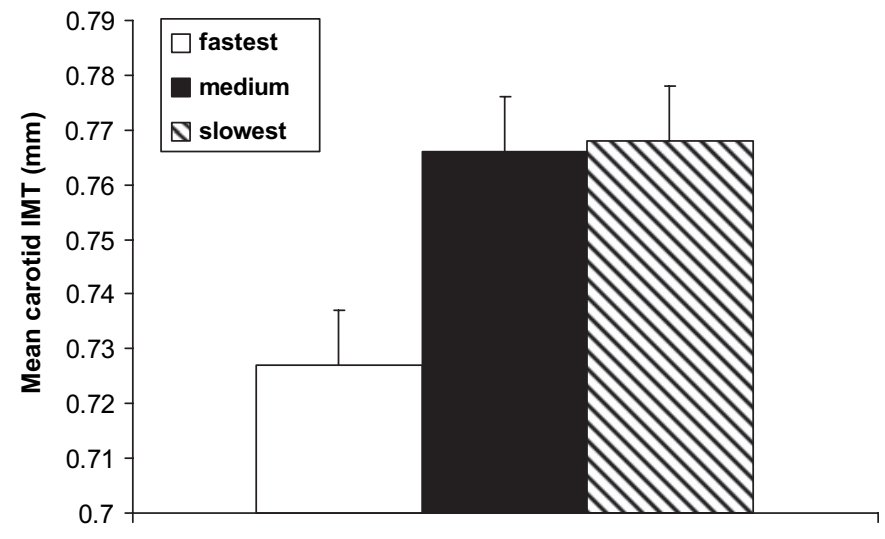

Figure 2 Association between carotid intima-media thickness (IMT) and objectively measured walking speed categorised into equal sexspecific tertiles. Values are mean $\pm S E M$, adjusted for age, sex, smoking, social status, systolic blood pressure, total and high-density lipoprotein cholesterol, C-reactive protein, fasting glucose, body mass index, shortform 36 score, statins.

studies of objective walking speed and CAC. In communitybased samples of healthy elderly participants, walking speed over a 15 -ft course was inversely related to CAC in women but not men, ${ }^{4}$ and maximum walking pace was inversely associated with carotid plaques and IMT. ${ }^{5}$ Our findings are consistent with the evidence that demonstrated associations of objective walking speed with incident CVD and total mortality. ${ }^{1}$ Other data have shown an association between impaired physical performance and inflammatory markers in the elderly. ${ }^{14}$

Studies examining the association between self-reported measures of physical activity and CAC have generally produced inconsistent findings, ${ }^{15-17}$ which may be explained by differences in physical activity assessment techniques between studies and limitations of self-reported instruments. In a recent study self-reported brisk walking pace was associated with lower CAC and IMT in men but only IMT in women, ${ }^{6}$ which contrasts with our results that showed no associations.

\section{MECHANISMS}

As the study was cross-sectional, we cannot determine the directionality of the association between walking speed and preclinical atherosclerosis. Slow walking speed is related to lack of physical exercise, which is a well-established CVD risk factor, ${ }^{18}$ and studies have demonstrated that physical inactivity in mid-life can predict functional decline in older age. ${ }^{19}$ Therefore, the inverse association of walking speed and subclinical atherosclerosis in older age may reflect cardioprotective effects of physical fitness and suggest that slow walking speed is an antecedent to CVD rather than a consequence. Recent data have confirmed strong inverse associations of exercise capacity, chronotropic response and abnormal heart recovery during exercise stress testing with CAC burden. ${ }^{20}$ Given that our objective measure of walking speed was closely associated with selfreported usual walking pace, this might reflect a simple objective measure of habitual physical activity and fitness. Nevertheless, self-reported walking pace in itself was not a robust predictor of subclinical atherosclerosis. Controlled training studies in elderly participants have demonstrated improvements in various metabolic risk factors, including inflammatory markers and insulin sensitivity. ${ }^{21} 22$ However, experimental studies that examine the effects of improved physical function on markers of subclinical atherosclerosis are required.
Alternatively, subclinical vascular factors may play a role in motor function, with slow walking speed reflecting functional consequences of underlying long-term conditions not yet apparent clinically. One hypothesis is that individuals with preclinical atherosclerosis have increased cerebral white matter hyperintensities, a condition that is associated with impaired physical function, including slower gait speed. ${ }^{23} 24$ This hypothesis would imply an association between walking speed and blood pressure, because hypertension, in addition to age, is the main risk factor for white matter hyperintensities. However, walking speed was not associated with systolic blood pressure and was only marginally associated with self-reported physical function in the present study. In addition, high walking variability has been associated with cognitive and functional status and subclinical brain vascular abnormalities, ${ }^{25}{ }^{26}$ although walking variability was not a predictor of subclinical atherosclerosis in the present study. Nevertheless, other important mechanisms may also be involved. For example, cardiac abnormalities such as impaired left ventricular function that has been associated with subclinical atherosclerosis, ${ }^{27}$ may partly explain reduced physical capacity. ${ }^{28}$ Subclinical disease of the blood vessels in the legs might lead to discomfort when walking, even though claudication is not present, resulting in a slower pace. Since the walking test is conducted from a standing start this may be another important aspect that is not captured in walking speed per se. This might partly explain the weak associations with self-reported walking pace becausee it does not take into account factors such as the initiation of movement.

\section{CONCLUSION}

The present findings have demonstrated the value of a simple short-distance walking test in capturing the severity of subclinical conditions in older adults not apparent in measures of clinical CVD. We measured walking speed with an 8-ft test in contrast to other studies that have generally employed longer walking distances ${ }^{1}$ or assessed maximal walking pace. ${ }^{5}$ The main difference in using a short-distance walking test is that it probably does not reflect aerobic capacity and the advantage is that it requires little space and is safe and easy to administer. The shortdistance test is thus able to detect differences in subclinical atherosclerosis and is also highly practical, making it a potentially attractive measure for office-based use in clinical practice.

Acknowledgements The authors acknowledge the contributions of the Whitehall II team to data collection, the staff of the Cardiac Imaging and Research Centre, Wellington Hospital, London, for performing electron beam computed tomography scans and the Vascular Physiology Unit, University College London, for performing IMT measures.

Funding This research was supported by the British Heart Foundation, UK (RG 05/006).

Competing interests None.

Ethics approval This study was conducted with the approval of the University College London Hospital committee on the ethics of human research.

Patient consent Obtained

Provenance and peer review Not commissioned; externally peer reviewed.

\section{REFERENCES}

1. Newman AB, Simonsick EM, Naydeck BL, et al. Association of long-distance corridor walk performance with mortality, cardiovascular disease, mobility limitation, and disability. JAMA 2006;295:2018-26.

2. Greenland $\mathbf{P}$, Bonow RO, Brundage $\mathrm{BH}$, et al. American College of Cardiology Foundation Clinical Expert Consensus Task Force. ACCF/AHA 2007 clinical expert consensus document on coronary artery calcium scoring by computed tomography in global cardiovascular risk assessment and in evaluation of patients with chest pain: a report of the American College of Cardiology Foundation Clinical Expert Consensus Task Force developed in collaboration with the Society of Atherosclerosis Imaging and 
Prevention and the Society of Cardiovascular Computed Tomography. J Am Coll Cardiol 2007:49:378-402.

3. Halcox JP, Donald AE, Ellins $E$, et al. Endothelial function predicts progression of carotid intima-media thickness. Circulation 2009;119:1005-12.

4. Inzitari M, Naydeck BL, Newman AB. Coronary artery calcium and physical function in older adults: the cardiovascular health study. J Gerontol A Biol Sci Med Sci 2008;63:1112-18.

5. Elbaz A, Ripert M, Tavernier B, et al. Common carotid artery intima-media thickness, carotid plaques, and walking speed. Stroke 2005:36:2198-202.

6. Bertoni AG, Whitt-Glover MC, Chung H, et al. The association between physical activity and subclinical atherosclerosis: the Multi-Ethnic Study of Atherosclerosis. Am J Epidemiol 2009;169:444-54.

7. Marmot MG, Brunner E. Cohort profile: the Whitehall II study. Int J Epidemiol 2005;34:251-6.

8. Guralnik JM, Simonsick EM, Ferrucci L, et al. A short physical performance battery assessing lower extremity function: association with self-reported disability and prediction of mortality and nursing home admission. J Gerontol 1994:49:M85-94.

9. Singh-Manoux A, Hillsdon M, Brunner E, et al. Effects of physical activity on cognitive functioning in middle age: evidence from the Whitehall II prospective cohort study. Am J Public Health 2005;95:2252-8.

10. Ware JE. Measuring patient's views: the optimum outcome measure. SF36: a valid, reliable assessment of health from the patient's point of view. BMJ 1993:306:1429-40.

11. Anand DV, Lim E, Darko D, et al. Determinants of progression of coronary artery calcification in type 2 diabetes role of glycemic control and inflammatory/vascular calcification markers. J Am Coll Cardiol 2007;50:2218-25.

12. Arad $\mathbf{Y}$, Goodman $\mathrm{KJ}$, Roth $\mathrm{M}$, et al. Coronary calcification, coronary disease risk factors, C-reactive protein, and atherosclerotic cardiovascular disease events: the St. Francis Heart Study. J Am Coll Cardiol 2005;46:158-65.

13. Brunner E, Shipley M, Spencer V, et al. Social inequality in walking speed in early old age in the Whitehall II Study. J Gerontol A Biol Sci Med Sci 2009 [Epub ahead of print].

14. Cesari M, Penninx BW, Pahor M, et al. Inflammatory markers and physical performance in older persons: the InCHIANTI study. J Gerontol A Biol Sci Med Sci 2004;59:242-8.

15. Desai MY, Nasir K, Rumberger JA, et al. Relation of degree of physical activity to coronary artery calcium score in asymptomatic individuals with multiple metabolic risk factors. Am J Cardiol 2004;94:729-32
16. Folsom AR, Evans GW, Carr JJ, et al. Association of traditional and non traditional cardiovascular risk factors with coronary artery calcification. Angiology 2004;55:613-23.

17. Taylor AJ, Watkins T, Bell D, et al. Physical activity and the presence and extent of calcified coronary atherosclerosis. Med Sci Sports Exerc 2002;34:228-33.

18. Davey Smith G, Shipley MJ, Batty GD, et al. Physical activity and cause-specific mortality in the Whitehall study. Public Health 2000;114:308-15.

19. Lang IA, Guralnik JM, Melzer D. Physical activity in middle-aged adults reduces risks of functional impairment independent of its effect on weight. J Am Geriatr Soc 2007; 55:1836-41.

20. Möhlenkamp S, Lehmann N, Schmermund A, et al. on behalf of the Heinz Nixdorf Recall Study Investigators. Association of exercise capacity and the heart rate profile during exercise stress testing with subclinical coronary atherosclerosis: data from the Heinz Nixdorf Recall study. Clin Res Cardiol 2009;98:665-76.

21. Kohut ML, McCann DA, Russell DW, et al. Aerobic exercise, but not flexibility/ resistance exercise, reduces serum IL-18, CRP, and IL-6 independent of beta-blockers, BMI, and psychosocial factors in older adults. Brain Behav Immun 2006:20:201-9.

22. Brooks N, Layne JE, Gordon PL, et al. Strength training improves muscle quality and insulin sensitivity in Hispanic older adults with type 2 diabetes. Int J Med Sci 2006;18:19-27.

23. Longstreth WT Jr, Manolio TA, Arnold A, et al. Clinical correlates of white matter findings on cranial magnetic resonance imaging of 3301 elderly people. The Cardiovascular Health Study. Stroke 1996;27:1274-82.

24. Whitman GT, Tang $Y$, Lin A, et al. A prospective study of cerebral white matter abnormalities in older people with gait dysfunction. Neurology 2001;57:990-4.

25. Rosano C, Brach J, Studenski S, et al. Gait variability is associated with subclinical brain vascular abnormalities in high-functioning older adults. Neuroepidemiology 2007:29:193-200.

26. Brach JS, Studenski SA, Perera S, et al. Gait variability and the risk of incident mobility disability in community-dwelling older adults. J Gerontol A Biol Sci Med Sci 2007:62:983-8.

27. Edvardsen T, Detrano R, Rosen BD, et al. Coronary artery atherosclerosis is related to reduced regional left ventricular function in individuals without history of clinical cardiovascular disease: the Multiethnic Study of Atherosclerosis. Arterioscler Thromb Vasc Biol 2006;26:206-11.

28. Newman AB, Gottdiener JS, McBurnie MA, et al. Cardiovascular Health Study Research Group. Associations of subclinical cardiovascular disease with frailty. J Gerontol A Biol Sci Med Sci 2001;56:M158-66. 